\title{
Consciousness and the prefrontal parietal network: insights from attention, working memory, and chunking
}

\author{
Daniel Bor ${ }^{1,2 *}$ and Anil K. Seth ${ }^{1,2}$ \\ 1 Sackler Centre for Consciousness Science, University of Sussex, Brighton, UK \\ ${ }^{2}$ Department of Informatics, University of Sussex, Brighton, UK
}

Edited by:

Naotsugu Tsuchiya, Monash

University, Australia

Reviewed by:

Ryota Kanai, University College London, UK

Guido Hesselmann, Charité Campus

Mitte, Germany

${ }^{*}$ Correspondence:

Daniel Bor, Sackler Centre for

Consciousness Science, Department of Informatics, University of Sussex,

Brighton BN1 90J, UK

e-mail:d.bor@sussex.ac.uk
Consciousness has of late become a "hot topic" in neuroscience. Empirical work has centered on identifying potential neural correlates of consciousness (NCCs), with a converging view that the prefrontal parietal network (PPN) is closely associated with this process. Theoretical work has primarily sought to explain how informational properties of this cortical network could account for phenomenal properties of consciousness. However, both empirical and theoretical research has given less focus to the psychological features that may account for the NCCs. The PPN has also been heavily linked with cognitive processes, such as attention. We describe how this literature is under-appreciated in consciousness science, in part due to the increasingly entrenched assumption of a strong dissociation between attention and consciousness. We argue instead that there is more common ground between attention and consciousness than is usually emphasized: although objects can under certain circumstances be attended to in the absence of conscious access, attention as a content selection and boosting mechanism is an important and necessary aspect of consciousness. Like attention, working memory and executive control involve the interlinking of multiple mental objects and have also been closely associated with the PPN. We propose that this set of cognitive functions, in concert with attention, make up the core psychological components of consciousness. One related process, chunking, exploits logical or mnemonic redundancies in a dataset so that it can be recoded and a given task optimized. Chunking has been shown to activate PPN particularly robustly, even compared with other cognitively demanding tasks, such as working memory or mental arithmetic. It is therefore possible that chunking, as a tool to detect useful patterns within an integrated set of intensely processed (attended) information, has a central role to play in consciousness. Following on from this, we suggest that a key evolutionary purpose of consciousness may be to provide innovative solutions to complex or novel problems.

Keywords: consciousness, prefrontal cortex, parietal cortex, attention, working memory, chunking, theory

\section{INTRODUCTION}

Questions concerning the nature of consciousness have profound and widespread personal, ethical and clinical implications, and a comprehensive understanding of consciousness is one of the most important and pressing issues in biology (Seth, 2010). Although consciousness is difficult to define (Zeman, 2005), we take the base concept to be the presence of phenomenal content for an agent, and we emphasize a distinction between conscious level (i.e., a position on a scale from total unconsciousness as in coma to vivid and alert wakefulness) and conscious content (i.e., the components of any given conscious scene - the qualia; Seth et al., 2008). We assume that a non-zero conscious level is needed for any conscious contents to exist.

In recent years, the scientific study of human consciousness has been transformed from a niche field into an increasingly popular, active, and sophisticated topic of research. At the level of brain mechanisms, consciousness science now synthesizes results from a broad range of techniques, including electrophysiology, functional magnetic resonance imaging (fMRI), magneto/electroencephalography (M/EEG), intracranial recordings, neuropsychology, and computational models (Tononi and Koch, 2008). In the next section, we will describe how a coherent pattern has emerged from these studies, in which two tiers of brain regions seem critical for consciousness: first, modality specific regions, such as those in extra-striate cortex, underlie the specific (phenomenal) contents of consciousness; second, the prefrontal parietal network (PPN) in concert with the thalamus is heavily implicated in consciousness more generally, regardless of content. The PPN can therefore be viewed as a "core correlate" of consciousness.

Although some theories of consciousness have been predicated on the link between consciousness and the PPN, others are more neutral on this issue. In the section "Theories of Consciousness," we will outline the main theories of consciousness and discuss the extent these theories are consistent with the empirical data linking consciousness with the PPN. We will also describe the psychological components of these theories, while noting that detail is currently lacking on this front. Instead, theoretical emphasis is 
given to widespread cortical network activity as a necessary component of consciousness, which is commonly discussed in terms of integrated information.

One approach to add psychological detail to models of consciousness would be to examine what other functions the PPN has been associated with. Fortunately, the PPN is one of the most studied of brain networks, and - beyond the context of consciousness science per se-has been closely linked with attention, among other functions (Duncan, 2006; Bor and Owen, 2007). In the section "The Overlap Between Attention and Consciousness," we critically assess the increasingly popular view of the independence of conscious and attentional processes (van Boxtel et al., 2010b). In contrast, we will argue that all conscious events require attention in some form, and that attention has a critical role to play in selecting conscious contents.

The PPN is closely associated with a far broader set of cognitive functions than merely attention (Duncan, 2006; Bor and Owen, 2007). For instance, working memory, executive control, and chunking have been particularly robustly associated with the PPN (Bor et al., 2003, 2004; Abe et al., 2007; Bor and Owen, 2007; Erickson et al., 2007; Koechlin and Summerfield, 2007). In the section "What is the Functional Role of the Prefrontal Parietal Network?" we outline how this range of cognitive processes may furnish models of consciousness with further psychological detail. We view consciousness as serving to provide innovative solutions to otherwise intransigent problems: we suggest that consciousness involves attending to information relevant to a biological goal, especially when automatic unconscious routines are unable to carry out that goal. Typically, this will occur for novel or complex tasks, where multiple features need to be managed simultaneously or in series, via working memory. We argue that chunking may play a special role in consciousness, by detecting and encoding regularities between items in working memory, thus aiding understanding, so that a given goal is more likely to be reached and automation accelerated.

\section{EVIDENCE LINKING THE PREFRONTAL PARIETAL NETWORK WITH CONSCIOUS CONTENT}

Although studies have reported PPN activity during subliminal processing (Diaz and McCarthy, 2007; Luo et al., 2009), the overwhelming evidence, both from lesion and perturbation studies and functional imaging studies, suggests that PPN is very closely associated with consciousness.

\section{LESION AND PERTURBATION STUDIES OF CONSCIOUS CONTENT}

Both focal lesion patient studies and transcranial magnetic stimulation (TMS) experiments in normal volunteers strongly implicate the PPN in supporting conscious contents. For instance, in one study patients with unilateral prefrontal cortex (PFC) lesions and healthy controls were briefly shown a number at one of four locations, followed after a variable delay by a mask (Del Cul et al., 2009). Subjects stated whether or not they had seen the number, and then guessed what the number was. Although objective performance for seen trials was matched between patients and controls, PFC patients were significantly less likely to consciously detect trials answered correctly, compared to controls.
Though conscious contents were not abolished in these cases, more severe consciousness impairments following unilateral PFC damage may be limited by extensive plasticity in these regions, such that the undamaged hemisphere can rapidly take over function (Voytek et al., 2010). Knight and Grabowecky (1995) have described a rare bilateral PFC lesion patient who was awake, but was otherwise almost entirely unresponsive to stimuli. With respect to conscious level rather than content, bilateral diffuse damage to prefrontal and parietal white matter is associated with vegetative state or coma (Tshibanda et al., 2009), while restoration of functional connectivity between the thalamus and PFC, as well as the anterior cingulate, is associated with recovery from vegetative state (Laureys et al., 2000).

Parietal lesions also lead to impaired conscious processing. For instance, Simons and colleagues compared bilateral posterior parietal lesion patients and controls on a long-term memory task, involving the recollection of the context in which stimuli are first encountered. Although recollection performance was matched between groups, the parietal lesion group exhibited impaired subjective experience of the memories they were retrieving (Simons et al., 2010).

Complementing the patient data, studies in normal volunteers using TMS have strongly implicated the PPN in consciousness. For instance, Turatto and colleagues presented volunteers with two grids of four faces for $200 \mathrm{~ms}$, separated by a $300 \mathrm{~ms}$ blank screen. On half the trials, the two sets of faces were non-identical. When a repetitive train of eight TMS pulses at $10 \mathrm{~Hz}$ was administered to right dorsolateral prefrontal cortex (DLPFC) at the onset of the first grid, the participants' ability to detect the change was impaired (Turatto et al., 2004). A further study by Beck et al. (2006) using a very similar paradigm, demonstrated that change detection is also impaired following TMS to the right posterior parietal cortex. A more recent study used a novel TMS technique, known as continuous theta burst stimulation (cTBS), which involves a rapid train of TMS pulses, for approximately $40 \mathrm{~s}$, so that activity in the stimulated region is suppressed for a sustained period of time, approximately $30 \mathrm{~min}$ in length (Huang et al., 2005). Rounis et al. (2010) gave participants a masked low contrast visual detect task both before and after TMS administration bilaterally to the DLPFC. Although objective performance was titrated to $75 \%$ accuracy on both occasions, impaired visibility ratings were found during the block following TMS. Another cTBS study by Kanai et al. (2010) reported that TMS applied to posterior parietal cortex increased the perceptual duration prior to a switch in a motioninduced bistable visual stimulus. Although bilateral results were found here, Zaretskaya et al. (2010), using a similar paradigm, found results somewhat limited to the right intraparietal sulcus, although considerable individual differences were reported.

This tendency in the literature to show preferential right PPN involvement (Turatto et al., 2004; Beck et al., 2006; Zaretskaya et al., 2010) echoes findings from the attentional neurological condition, hemispatial neglect, which in the vast majority of cases follows right PPN damage (Husain and Kennard, 1996; Husain and Rorden, 2003). In addition, the parietal region in these TMS studies is very close to that associated in functional imaging studies with attentional switching processes (Corbetta et al., 1995; Yantis et al., 2002). Indeed, both Kanai and Zaretskaya posit that the 
mechanism for delays in perceptual switches as a result of TMS to posterior parietal cortex is due to a reduction in attentional resources. In line with this, a similar reduction in perceptual switch rate to binocular rivalry stimuli can be induced just by diverting attention away from the bistable stimuli (Paffen et al., 2006; Alais et al., 2010). Furthermore, by indirectly inferring attention state from EEG signatures, Zhang et al. (2011) demonstrated that diverting attention away from a binocular rivalry stimulus abolishes rivalry, suggesting that not only does attention modulate binocular rivalry rates, but that it is a necessary component of the phenomenon.

The lesion and perturbation studies described above strongly implicate all key individual components of the PPN in conscious processing. However, such methods have a more limited role in examining the relationship between consciousness and the PPN as a whole. Functional neuroimaging studies, discussed next, are well placed to address this question.

\section{FUNCTIONAL IMAGING STUDIES}

In one of the first studies to examine visual consciousness using fMRI, only PPN and extra-striate activity were observed when switches in visual consciousness in a binocular rivalry paradigm were compared with a perceptually matched condition without rivalry (Lumer et al., 1998). A follow-up binocular rivalry study without behavioral responses demonstrated that motor output cannot be responsible for this pattern of activity: extra-striate activity reflecting changes in perception most closely correlated with PPN activity, whereas V1 activity failed convincingly to correlate with any other region (Lumer and Rees, 1999). The PPN has also been associated with consciousness in fMRI studies of other bistable paradigms, such as ambiguous figures (Kleinschmidt et al., 1998), the spinning wheel illusion (Sterzer et al., 2002), and flicker (Carmel et al., 2006).

It is unclear, however, whether such PPN activity reflects the cause of changes in conscious contents or alternatively the downstream effects of these changes. Knapen et al. (2011) have recently provided evidence that PPN activity, at least in the right hemisphere, is a response to perceptual change in binocular rivalry: normal participants were presented with a range of binocular rivalry stimuli, which induced variable transition periods between the two competing percepts. Longer transitions were associated with prolonged right PPN activity. Simulated external transitions, which carefully matched internal transition lengths, were associated with similar patterns of PPN activity, suggesting that the PPN was mainly associated with a response to perceptual change. Consistent with this view, fMRI responses correlating with the percept have been found in early sensory areas in many bistable paradigms (Lee et al., 2005), and in binocular rivalry even as early as the lateral geniculate nucleus (LGN) in the thalamus (Haynes et al., 2005). Sillito et al. (2006), however, stress the importance of cortical outputs to LGN, suggesting either that LGN perceptual-tracking activity has a cortical source, possibly in V1 or MT, or that even if LGN activity is the cause of binocular rivalry, that there are clear cortical pathways which could modulate various features of the binocular rivalry percept by differential attentional focus. Summarizing these observations, although the causal factors underlying bistable perceptual transitions remain unclear, there is little doubt that PPN activity reflects the resulting subjective conscious contents.
Another relevant class of fMRI studies utilize psychophysical masking paradigms in order to compare visible with invisible stimuli. For instance, Dehaene et al. (2001) showed that when visibility of word stimuli was modulated by masking, those stimuli which were consciously perceived additionally activated modality specific regions in fusiform gyrus, as well as inferior frontal and parietal regions. However, a general problem with masking paradigms is that they may confound conscious contents with objective performance. Addressing this concern, Lau and Passingham (2006) implemented a metacontrast masking paradigm, which ensured that objective performance was equated while subjective visibility could be manipulated. In this more controlled design, only the DLPFC portion of PPN was reported to covary with visibility.

Electroencephalography studies have also implicated the PPN in consciousness. Melloni and colleagues compared oscillatory EEG activity evoked by visible and invisible words in a delayed matching to sample task. Consistent with theories emphasizing the importance of functional connectivity in consciousness (see the next section), they found that both visible and invisible words caused increases in local gamma-band oscillations, but only visible words induced increased long-range gamma-band synchronization, consistent with PPN involvement. In addition, during the maintenance period, enhanced theta oscillations were observed over frontal regions (Melloni et al., 2007). More direct evidence is provided by a recent study by Hipp et al. (2011) in which EEG synchrony was assessed between source-localized cortical regions; the authors identified two distinct cortical networks predictive of perception: a beta-band fronto-parieto-occipital network and a gamma-band centro-temporal network.

Conventional M/EEG studies carry inevitable ambiguities regarding spatial resolution and localization, and suffer poor signal-to-noise ratios. Recent studies using intracranial EEG measurements are therefore particularly valuable. In one landmark study, intracranially implanted presurgical patients were presented with invisible (masked) or visible (unmasked) words. Sustained consciousness-related activity for the unmasked words was found most robustly in PFC (Gaillard et al., 2009).

While all studies described so far have examined visual consciousness, similar results have been reported in other modalities. For instance, Sadaghiani et al. (2009) used an auditory detection paradigm at threshold to show that conscious detection reflected prefrontal and inferior parietal activity, though other regions were also found, including the insula, thalamus, and striatum. In another intriguing study, Hasson et al. (2007) used a passive cross-modal paradigm involving the McGurk effect, in which the auditory perception of a given syllable is determined by the fusion of two different auditory and visual syllables. Using repetition priming, participants were played an audiovisual target that was preceded either by a stimulus that matched the target's visual features alone, its auditory features alone, or neither, but which instead matched the subjective percept of the fusion of the target's competing auditory and visual syllables. While auditory cortex regions tracked the objective features of the stimulus, PPN activity was associated with the conscious percept, as reflected in a reduction in activity limited to the fused prime trials.

The studies described so far have examined the neural correlates of changes in consciousness. Other experiments have shown that sustaining a percept also implicates the PPN. For instance, 
Erikkson and colleagues presented participants in the fMRI scanner with fragmentary figures, which unlike bistable viewing paradigms, involve only a single perceptual transition, followed by a period of sustained perception. The PPN was found to be associated both with transient and sustained aspects of visual perception on this task (Eriksson et al., 2004).

In addition to functional imaging studies, two recent structural imaging studies have associated the PPN with consciousness. First, Kanai et al. (2010) demonstrated that individual differences in posterior parietal cortex cortical thickness, gray matter density, and white matter integrity correlate with volunteers' alternation rates when viewing bistable figures. Second, Fleming and colleagues used structural imaging to examine individual differences in metacognitive ability. Metacognitive ability is a particularly stringent test of consciousness, since it explicitly focuses on conscious report, thus avoiding the association in some other studies between neural signatures and responses which may be correct either by chance or due to unconscious knowledge (Lau and Rosenthal, 2011). Fleming and colleagues presented volunteers with two sets of six Gabor patches, with one set of six including a single Gabor patch with a marginally higher contrast. Participants made a judgment as to which set included the deviant patch and then made a confidence report on their previous decision. Using a staircase function, objective performance was fixed at 71\%, but volunteers' ability to match confidence levels with performance (metacognitive ability) differed markedly. Individual differences in volunteers' metacognitive ability correlated both with prefrontal gray matter volume and white matter integrity (Fleming et al., 2010).

The association between PPN and conscious content could be further tested by reducing conscious level, thus also diminishing the capacity for conscious processing, and observing whether PPN activity falls in parallel. Complementing the data from coma and vegetative state patients mentioned above, Davis et al. (2007) have shown that parts of the PPN appear necessary for normal levels of consciousness. In this study the general anesthetic, propofol, was administered to normal participants at various intensities, while they were presented with auditory stimuli at multiple levels of complexity. Lateral prefrontal activity reduced in line with a reduction in conscious level, and reflected an impairment in sentence comprehension and subsequent recall.

In summary, PPN lesions impair normal conscious function, as does TMS when applied to these regions. Functional and structural imaging studies have strongly implicated the PPN in perceptual transitions, the conscious detection of stimuli in a range of modalities, sustaining percepts, and in metacognitive decisions on those percepts. Finally, a reduction of conscious level when under general anesthesia is associated with a reduced lateral prefrontal activity.

\section{THEORIES OF CONSCIOUSNESS NEURAL THEORIES OF CONSCIOUSNESS AND THE PREFRONTAL PARIETAL NETWORK}

A growing set of consciousness theories have attempted to link experimental findings in consciousness science to cognitive and neurophysiological architectures (see Seth, 2007; Kouider, 2009 for reviews): prominent examples of theories articulated at the neural level include (neuronal) global workspace theory (Baars, 1988, 2005; Dehaene and Naccache, 2001; Baars et al., 2003; Dehaene and
Changeux, 2011), reentry theory (Lamme, 2006, 2010), dynamic core, integrated information theories (Tononi and Edelman, 1998; Edelman, 2003; Balduzzi and Tononi, 2008; Tononi, 2008), and causal density theory (Seth et al., 2011).

Neuronal global workspace theory is the most explicit in terms of PPN activity (Dehaene and Naccache, 2001; Dehaene and Changeux, 2011). According to this theory, conscious content, which is determined by domain-specific local processing, gains access to consciousness via integration into a "global workspace." This integration is mediated via long-range corticocortical "workspace" neuronal projections that are particularly dense in prefrontal, parietal, and cingulate regions. A specific conscious content is suggested to be encoded by the sustained activity of a fraction of these workspace neurons, the rest being inhibited (Dehaene and Changeux, 2011). Other key elements of the theory propose that conscious contents are determined in an "all or none" fashion via "ignition" of the global workspace, where ignition is reflected in late cortical potentials distributed across PFC and other high-level associative cortices, and in high frequency (e.g., gamma-band) increases in power and synchrony. Interestingly, while this theory is explicit about neurobiological substrates, it leaves behind a substantial portion of the psychological detail that was part of the original "cognitive" global workspace theory introduced by Baars (1988). We return to Baars' work in the following section.

Lamme's "neural stance" on consciousness identifies reentrant or feedback connections as the key ingredient in generating conscious content (Lamme, 2006, 2010). Synthesizing work from a large number of studies examining neural responses to masked and unmasked stimuli, Lamme proposes that reentrant connections encapsulating the PPN and lower-level modality specific cortices are necessary for cognitively accessible, subjectively reportable conscious contents. This claim is compatible with neuronal global workspace theory; the theories further align in associating late components of cortical evoked potentials with cognitive access. The theories diverge, however, with Lamme's claim that a nonaccessible form of conscious content (i.e., phenomenal consciousness without access consciousness, in the terminology of Block, 2007), is generated by reentry locally within posterior cortical regions. A major challenge attending this claim is of course how to verify the existence of such conscious contents in the absence of verbal or behavioral report; a lively debate continues around this issue (Block, 2007; Kouider et al., 2010; Lamme, 2010; Dehaene and Changeux, 2011).

Zeki (2007) has proposed a related theory, microconsciousness, which is perhaps the most ambivalent of modern theories toward the association between the PPN and consciousness. While Zeki concedes that unified forms of consciousness or those which involve access may require the PPN, he believes, like Lamme, that there is a non-reportable, purely phenomenal form of consciousness, which can be supported by local sensory regions, for instance with visual cortex representing visual consciousness. The theory of micro-consciousness diverges from Lamme's reentrant theory, however, in that for Zeki consciousness is not dependent on feedback pathways, for instance from V4 to V1 for color vision. Instead, it is suggested that the level of consciousness for a given form of phenomenal content is dependent only 
on the strength of activity within the region that processes that form of information; therefore, V4 alone is capable of generating color vision, for instance. Indeed, patients with focal lesions in V1 still report significant, though degraded, visual phenomenology, despite non-functional back-projections (Ffytche and Zeki, 2011).

Although much evidence has already been cited in the section "Evidence Linking the Prefrontal Parietal Network with Conscious Content" to demonstrate the importance of the PPN for consciousness, Fisch et al. (2009) have reported indirect evidence in support of micro-consciousness. Using intracranial electrodes on a wide range of cortical surface locations in 11 patients, a backward masking paradigm was employed, where pictures of various categories were briefly presented, followed by a mask. Localized bursts of gamma-band activity were found, primarily in visual regions, and with an early time signature approximately 150-200 ms following stimulus onset. The authors note, however, that this doesn't rule out a critical role of the PPN for conscious recognition in a later time window (>300 ms). Indeed, such activity was observed in the same study.

The dynamic core, integrated information, and causal density theories are also compatible with a key role of the PPN in consciousness, though these theories are less anatomically specific than those discussed above. The dynamic core theory (Tononi and Edelman, 1998; Edelman, 2003) proposes that consciousness depends on a flexible and dynamically constituted functional cluster of thalamocortical neurons, generating dynamics characterized by conjoined functional integration and functional segregation. According to the theory, these dynamics are mediated by highly reentrant connections within the thalamocortical system. The anatomical boundaries of the dynamic core are considered to be highly labile, so that neuronal groups or even brain regions may form part of the neural correlates of consciousness (NCC) at one time, but not at another. Having said this, the dynamic core theory may be considered an extension of Edelman's theory of neuronal group selection (TNGS) which proposes explicitly that consciousness emerges from interactions between posterior regions involved in perceptual categorization and frontoparietal regions implicated in the formation and maintenance of value-category memories (Edelman, 1989, 2003; Edelman et al., 2011). Taken together, these theories imply a core role for the PPN in generating conscious contents.

The information integration theory of consciousness (IITC; Balduzzi and Tononi, 2008; Tononi, 2008; Barrett and Seth, 2011) shares with the dynamic core theory an emphasis on quantitative description of conjoined segregation and integration in dynamics. The IITC operationalizes this notion in a subtly but significantly different way, as the extent to which a network generates "integrated information." In this view, "information" refers to how much the present state of a system reveals about its past state (or states), in terms of reduction of uncertainty or entropy; this aspect reflects the diversity and informativeness of conscious scenes. "Integration" reflects the degree to which this information is generated only by considering the system as a whole, over and above that generated by its parts considered independently; this aspect is argued to reflect the phenomenal unity of conscious scenes. Quantitative measures of integrated information, denoted by $\Phi$, have been proposed both by Tononi (Balduzzi and Tononi,
2008; Tononi, 2008) and by Barrett and Seth (2011); see Seth et al. (2011) for a summary.

Tononi emphasizes that the IITC accounts for some broad anatomical facts about consciousness, including its association with the thalamocortical system. However, besides noting that general architectural features of thalamocortical and corticocortical circuits (described further below) are compatible with generating high values of integrated information, the theory does not propose specific roles for the PPN with respect to these features (Tononi and Koch, 2008). Indeed, for Tononi, the IITC is strictly agnostic about implementation; consciousness could be attributed to any system generating sufficiently high-levels of integrated information. There are however weaker interpretations of the IITC which view integrated information as an "explanatory correlate" mapping features of phenomenology onto features of neural dynamics (Seth, 2009; Barrett and Seth, 2011); on this view, high integrated information may be necessary but not sufficient for consciousness, and incorporation of the PPN may therefore also become a necessary feature of the neural grounding of the theory.

A similar perspective applies to causal density theory, where "causal density" provides a third means of quantifying conjoined dynamical segregation and integration (Seth, 2005; Seth et al., 2008, 2011). Causal density leverages time-series analysis methods (specifically Granger causality, which is a statistical measure of causality based on relative predictability) to quantify the overall level of causal interactivity within a system. High values of causal density are obtained when system elements combine statistical independence (so that they provide potentially useful predictive information) with statistical dependence (so that this predictive information is in fact useful). Causal density is offered explicitly as an "explanatory correlate" (Seth, 2009) and as a necessary but not sufficient criterion for consciousness. As with $\Phi$, causal density may be compatible with general anatomical properties of the PPN and associated brain networks.

An important challenge for future work is to compare the neural theories described above in the context of structural properties of brain networks. While all theories are broadly compatible with the high-levels of interconnectedness observed in both corticocortical and thalamocortical networks (though only trivially so for Zeki's theory), rapidly developing research on specific topological properties of these networks may allow greater discriminatory power and shed new light on the potential importance of the PPN for consciousness. For example, suggestive new findings, based on combining multiple anatomical tracing studies, identify a tightly integrated "core circuit" in the macaque brain including the PPN, with the PFC containing a very large proportion of topologically central subregions (Modha and Singh, 2010).

\section{COGNITIVE THEORIES OF CONSCIOUSNESS AND THE PREFRONTAL PARIETAL NETWORK}

As neural theories of consciousness make progress in connecting neural to phenomenal properties, they often seem do so at the expense of psychological or cognitive detail. However, cognitive theories of consciousness have a long history, extending back to when "consciousness" as an explanandum was not often referred to explicitly (see de Gardelle and Kouider, 2009 for a review). Thus, in early work and anticipating later workspace theories, 
Broadbent (1958) associated consciousness with a "limited capacity channel" involved in serial processing; this theory entailed a very close association between consciousness and attention. Some years later, Baddeley and Hitch (1974) associated conscious contents with working memory, moving yet closer toward central tenets of global workspace theory. Later cognitive theories made explicit reference to elements of the PPN. For example, Shallice identified consciousness indirectly with a "supervisory attentional system" situated in PFC, controlling the activity of "lower level" sensorimotor modules (Norman and Shallice, 1986). This theory operationalized consciousness as a form of executive control, without much reference to phenomenal aspects.

Building on much of this early work, the predominant cognitive theory of consciousness remains Bernard Baars' global workspace theory (Baars, 1988, 2005). The core elements of this theory are (i) competition among local (unconscious) processes for access to a "global workspace" the contents which are made globally available, (ii) "broadcast" of workspace contents to a broad repertoire of receiving modules. With these core elements, global workspace theory fluently accounts for the apparent seriality and unified nature of conscious scenes, as well as the parallel processing capacity of unconscious processes. As emphasized by Dehaene and Changeux (2011), the theory seems especially well suited to account for conscious access and is not normally interpreted as a theory of "phenomenal consciousness" of the form described by Block and Lamme [i.e., consciousness without cognitive access; (Block, 2007; Lamme, 2010)].

Efforts to ground global workspace theory in neurobiology (see Neural Theories of Consciousness and the PPN) have focused on these core elements (though "broadcast" remains poorly specified in neural terms) and have arguably come at the cost of neglecting some of the psychological detail embedded in its original cognitive instantiation. For example, Baars (1988) originally emphasized that the (conscious) contents of the global workspace could correspond, at any given time, to the contents of working memory; indeed the theory has recently been summarized as suggesting a "fleeting memory capacity that enables access between brain functions that are otherwise separate" (Baars, 2005). Cognitive, as opposed to neuronal global workspace theory also placed considerable emphasis on unconscious "contextual" systems which shape the conscious contents articulated within the workspace itself (Baars, 2005).

The "higher-order" thought (HOT) theory set forth most prominently by Rosenthal also deserves mention. On this theory, a mental state is conscious when a person is aware (or disposed toward being aware) of being in that state (Rosenthal, 2005). Theories differ according to whether awareness of the mental state is achieved by perceiving it (Lycan, 2004) or thinking about it (Rosenthal, 2005). Importantly, the theory is not circular; the higher-order thoughts/perceptions themselves are not consciously reportable unless accompanied by a corresponding third-order thought or perception. Although originally a philosophical theory, HOT theories have recently attracted attention within cognitive neuroscience. Lau has suggested that HOTs could be implemented as a process of internalized signal detection, in which perceptual signals are assessed for their reliability according to the precepts of signal detection theory. The neural mechanisms underlying this process are suggested to lie within DLPFC (Rounis et al., 2010; Lau and Rosenthal, 2011).

\section{THE OVERLAP BETWEEN ATTENTION AND CONSCIOUSNESS}

Before the connection between consciousness and the PPN was a focus of investigation, this network of brain regions was for many years associated with other cognitive processes, most notably working memory, executive functions, and attention (Cabeza and Nyberg, 2000). This overlap strongly suggests that these other cognitive processes are closely related to consciousness. In this section, we first examine the strength of the argument for treating attention and consciousness as independent processes, before outlining the common links between these two processes, as well as with related high-level cognitive functions.

\section{THE ARGUMENT FOR A DISSOCIATION BETWEEN ATTENTION AND CONSCIOUSNESS}

Even up to the start of the twenty-first century, many researchers took the view that attention and consciousness were putatively one and the same process, or that attention provided a "gateway" to consciousness (Broadbent, 1958; Posner, 1994; Rees and Lavie, 2001). For instance, Rees and Lavie (2001) noted the marked similarity in activation patterns between attention and consciousness studies and suggested that there is "a close functional relationship between attention and awareness." Over recent years, however, there has been a growing body of opinion that these two phenomena are highly or even entirely dissociable (Koch and Tsuchiya, 2007; van Boxtel et al., 2010a,b). For instance Koch and colleagues claim that attention and consciousness can be "manipulated independently" and that attention is neither necessary nor sufficient for consciousness (van Boxtel et al., 2010b).

The crux of this lively debate undoubtedly rests on questions of the definition and scope of attention and consciousness. In their latest review, van Boxtel et al. (2010b) limit attention to top-down voluntary attention, and define this as the main process by which an over-abundant data input is filtered into a much reduced set of salient items. They summarize attention as an "analyzer" of cognition, and consciousness as a "synthesizer." They operationally define consciousness as stimulus visibility.

In line with their position, there is good evidence that attentional processes, as defined above, can operate without the attended stimuli reaching consciousness (Bressan and Pizzighello, 2008; Kentridge et al., 2008; Bussche et al., 2010; Tapia et al., 2010). For example, Kentridge et al. (2008) demonstrated that attention can enhance the processing of unconscious targets: incongruent or congruent colored disk primes, made invisible by metacontrast masking, were followed by a subsequent visible colored annulus. When attention was directed toward the location of the invisible primed disks, the priming effect was enhanced.

Showing the opposite effect, Bressan and Pizzighello (2008) implemented a paradigm in which an invisible distracting moving stimulus nevertheless impaired accuracy on a primary task at fixation. The authors proposed that the distracting stimulus created a state of alertness and consequently a partial attentional shift away from the central task. In contrast, when the distracting stimulus was consciously detectable, accuracy was unimpaired, presumably 
because full processing allowed the designation of the distracting stimulus as irrelevant to the task, with minimal attentional resources attached to it. However, since this study is putatively governed by a bottom-up attentional process, it is not strictly relevant to the arguments of van Boxtel et al. (2010b). Nevertheless, it provides intriguing evidence that attention to unconscious stimuli can both enhance and interfere with a central conscious task.

Although such examples do indicate that attention is not sufficient for consciousness, it does not follow that attention is dissociated from consciousness. Indeed, the studies described above can be interpreted as showing that attention enhances existing conscious contents, or the probability that an item will gain access to consciousness. In Bressan and Pizzighello (2008), reduced performance on the primary task could be a consequence of reduced awareness of the target when attention is partly allocated elsewhere. Furthermore, in the study of Kentridge et al. (2008), improved target detection could reflect enhanced awareness following attentional boosting of congruent primes. In line with this position, attention has been shown to lower the threshold by which a stimulus is consciously detected, or enhance apparent contrast (Carrasco et al., 2000, 2004; see Figure 1 for illustration).

For evidence of a true (i.e., double) dissociation, what is required is a demonstration that increased attention for a specific visual feature leads to a reduction in the probability of conscious report of that feature. An apparently strong case for such a double dissociation was recently made by Koch and colleagues (van Boxtel et al., 2010a). A peripheral after-image inducer was made either visible or invisible, by continuous flash suppression. Attention was either directed to the inducer, or distracted by a central rapid serial visual presentation (RSVP) task. While increased visibility of the inducer also led to increased after-image duration, greater attention toward the inducer instead reduced after-image duration.
Although this pattern of results suggests a double dissociation between attention and consciousness, alternative interpretations do exist. For instance, it is possible that increased attention in this paradigm, rather than simply reducing the probability or duration of current conscious contents per se, instead provides a useful role in suppressing an unhelpful and aberrant after-image, so that other, more salient objects have a higher chance of gaining conscious access. Further experiments exploring double dissociations between consciousness and attention are needed, preferably in topics outside of visual illusory effects since such effects necessarily reflect idiosyncrasies of the visual system and may not directly reflect key mechanisms of consciousness itself.

Although the evidence that attention is not sufficient for consciousness is reasonably well established, the case for consciousness without attention is somewhat less clear. Evidence cited by van Boxtel et al. (2010b) for consciousness without attention mainly involves pop-out effects, gist effects, and detection of natural objects, such as animals and faces. In each of these examples, however, it is important to establish whether attention has been fully removed from the stimulus that the subject is conscious of, in terms of absence of both top-down attention and bottom-up attention. van Boxtel et al. (2010b) do not include or discuss bottom-up attentional effects, since they claim that bottom-up attention is necessarily engaged by visible moving stimuli, thus experimentally precluding the opportunity to dissociate these two processes. However, Bressan and Pizzighello (2008) findings suggest that bottom-up attention can be engaged by invisible stimuli and so there are not necessarily empirical barriers to including bottomup attention within a discussion of relations between attention and consciousness. Furthermore, by limiting their focus to studies manipulating only top-down effects, van Boxtel and colleagues still need to entertain the possibility that, for any evidence demonstrating consciousness without attention, bottom-up attentional

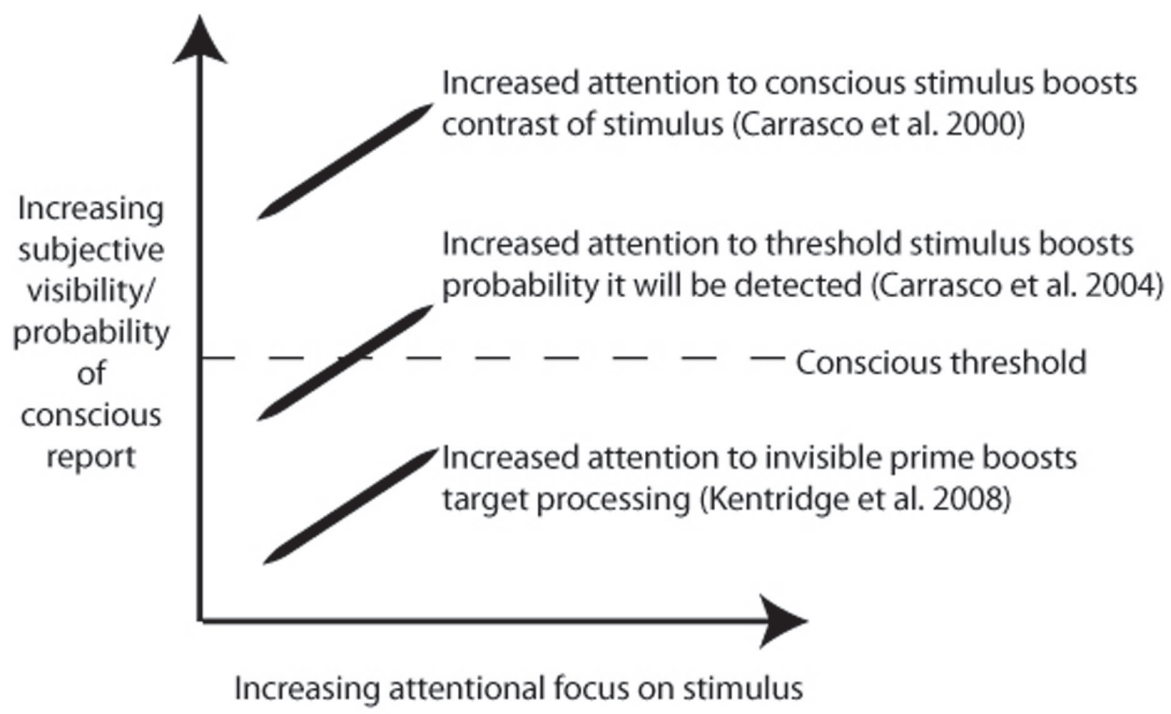

FIGURE 1 | Schematic illustration of possible relationships between attention and consciousness, with notable experimental examples. Note that although the lower example is taken by some as evidence for the independence of attention and consciousness, it is instead possible that in this and all other cases there is a strong positive correlation between attention and consciousness. 
processes may still be present and may be involved in selecting items for conscious access.

Taking each of the putative examples of consciousness without attention in turn: first, pop-out effects of primitive visual targets, for instance in a visual search task, have been assumed to occur preattentively. However, if attention is sufficiently engaged away from a simple target, such as a red element (with gray distracters) or a specific orientation (with orthogonally angled distracters), then there is a large detrimental effect on target detection (Joseph et al., 1997; Theeuwes et al., 1999). Furthermore, attention has been shown to modulate basic perceptual masking effects, such as object substitution (Tata and Giaschi, 2004) and metacontrast masking (Ramachandran and Cobb, 1995; Shelley-Tremblay and Mack, 1999; Boyer and Ro, 2007).

Second, natural scene perception, also known as "gist," is a process where various semantic and phenomenological features of a visual scene can be extracted, even with brief viewings of $150 \mathrm{~ms}$ (Thorpe et al., 1996). It has been assumed that the speed of this process precludes the possibility that selective attention plays a role, since selective attention requires at least $200 \mathrm{~ms}$ to become established. However, one recent study has shown that if attention is appropriately removed from a natural scene, then inattentional blindness for that scene ensues (Cohen et al., 2011). Using a dual task paradigm in a set of experiments, attention was withdrawn from the natural scene by employing multi-object tracking (MOT) or a RSVP task. Using MOT, failure immediately to perceive a visual scene occurred in $88 \%$ of participants, compared with only $4 \%$ when carrying out the single task of detecting and classifying the visual scenes. Similar results occurred for the RSVP task (77\% of participants were unable immediately to perceive the scene, compared with $7 \%$ for the detection and classification task alone). For both tasks, however, more participants were able to classify the scene on question probes following the trial. In follow-up experiments that modulated the difficulty of the MOT and RSVP tasks, those tasks that were more difficult and thus requiring more attentional resources further impaired conscious detection of the visual scene. This study clearly shows that a conscious sense of gist is dependent on having at least a minimal level of attentional processing available.

Third, and finally, the conscious detection of animals and faces is thought to have a privileged access due to its biological salience and can occur with very brief presentations, of the order of $20 \mathrm{~ms}$. There is some evidence that detection rates are unimpaired during a dual task, where attention is directed away from the naturalistic objects ( $\mathrm{Li}$ et al., 2002). However, these studies tend to present single stimuli in isolation. Attention might, to some degree, be automatically drawn to a single visually presented object, regardless of the inclusion of a distracting secondary task, especially if that object carries innate biological salience (such as a face), which would activate bottom-up attentional processes. Walker et al. (2008) sought to investigate the importance of this factor, by presenting animal images along with three other objects in a complex naturalistic scene. Using this approach, the dual task condition profoundly impaired detection of animal targets, even at long onset durations of approximately $500 \mathrm{~ms}$. In addition, Walker and colleagues found that even animal targets presented alone were detected less frequently under dual task than single task conditions, though to a lesser extent than when presented with the three distractors. Therefore, even the conscious detection of naturalistic objects is shown to be heavily modulated by attention, especially if complex scenes are involved.

As well as behavioral dissociations, neural dissociations between attention and consciousness have been proposed (Wyart and Tallon-Baudry, 2008; Tsubomi et al., 2011). For instance, Wyart and Tallon-Baudry (2008) used MEG while subjects either attended toward or away from a threshold stimulus, which was reported consciously $50 \%$ of the time. Independent of attention, consciously seen stimuli induced mid-gamma activity, whereas attention induced high-gamma activity independent of conscious report. However, since attention was manipulated by directing participants to a valid or invalid location, additional error monitoring processes for the attentional contrast may account for the dissociation. In addition, both the attentional and consciousness effects were largely confined to early visual regions, thus inviting further research to examine whether similar dissociations can be observed in regions more commonly and centrally associated with either attention or consciousness, such as the PPN.

A more recent study by Tsubomi et al. (2011) used fMRI to investigate similar possible dissociations. Subjects were presented with a visual backward masking flanker paradigm, in which attention to flankers enhanced target visibility and attention to the mask attenuated it. Attention to flankers compared to mask, where visibility was higher, was associated with increased occipito-temporal sulcus activity. In addition, for both attentional conditions, visibility was correlated with activity in the same sulcus. In contrast, PPN activity was observed for the two attentional conditions compared with a no-attend control, independent of target visibility. However, the extent to which this study was investigating conscious access may be limited, since almost all stimuli presented were sufficiently clear and visible for object identity to correctly occur. Indeed, if trials where subjects categorized the target as invisible were removed from the analysis, essentially the same pattern of results was found. Therefore, the observed occipito-temporal visibility effects may largely reflect the perceptual intensity of items that have already gained access to conscious.

In summary, although studies do report pop-out effects, gist processing and natural object detection occurring in the absence of attention, further research has shown that attentional resources are required in each case, in order to enable conscious report of target stimuli. Indeed, the fact that attention is required for popout effects of very simple visual stimuli, such as a colored element, or an oriented gabor patch (Joseph et al., 1997; Theeuwes et al., 1999) implies that for all conscious events, even those involving very primitive elements, at least some attentional resources are necessary. In addition, although a small number of studies have presented provisional evidence that neural dissociations can be found between attention and consciousness, this is in contrast to considerable evidence (see sections Evidence Linking the Prefrontal Parietal Network with Conscious Content above and What is the Functional Role of the Prefrontal Parietal Network? below) emphasizing a close neural overlap between these two processes. 
SHOULD ATTENTION INCLUDE WORKING MEMORY WITHIN ITS SCOPE? As mentioned above, the question of overlap between attention and consciousness critically depends on the corresponding definitions. If it is assumed, as argued above, that attention is necessary for consciousness, and thus may play an important role in its function, one useful approach would be to expand on the definition of attention.

One prominent model of attention is Bundesen's neural theory of visual attention (NTVA; Bundesen, 1990; Bundesen et al., 2005). In the NTVA model, two waves of processing occur. During the first wave, processing resources are distributed unselectively across the visual field. The product of this wave of processing is a saliency map, which stores the attentional weight for each object in the visual field, based on either matches with longterm memory items or biologically salient representations. The second wave of processing involves selective competition in the form of a race to populate visual short-term memory until capacity is reached (approximately four items), by the reallocation of neuronal resources for each object, according to the attentional weight assigned to it. Weighting for objects is governed by the number of neurons recruited to represent it, while weighting for a given object feature is controlled by the scaling of average neuronal activity. Both top-down and bottom-up activations are involved in assigning neuronal populations and activity.

Single neuron recording in monkeys has provided good support for the idea of a first, unselected wave of activation. For instance, Chelazzi et al. (1998) found using a visual search task, that in the first 150-200 ms following stimulus onset, monkey inferotemporal cortex neurons showed an equally raised response to any set of stimuli. Only after this stage do the neurons modulate their activity in line with the top-down goals of the task, so that neurons coding for the target continue to increase in firing rate, while those coding for non-targets rapidly reduce in activity.

One critical feature of NTVA, therefore, is that in the first wave of activity there is a positive, though weak and unbiased set of weightings for items in the visual scene, which could be thought of as an unselective attentional enhancement for the visual field. Intriguingly, this attentional feature could be a cognitive and neural implementation of "gist," discussed in the previous section, and may explain the extraction of some phenomenological and semantic features from a brief natural scene presentation, as well as the lack of detail of the percept. In addition, similar positive, though unselective attentional weightings could in principle explain other effects, such as iconic memory persistence and partial reportability. Indirect evidence in support of this has been reported in an fMRI study by Ruff et al. (2007), who presented volunteers with both a partial report and iconic memory task. Very similar attentional networks, especially comprising the PPN, were observed for both tasks.

Suggestively, NTVA includes working memory capacity as one important parameter, such that the outputs of attentional selection, via object classification from long-term memory, are placed in a limited capacity short-term memory store.

In another prominent recent attentional model, Knudsen agrees with van Boxtel and colleagues that attention is responsible for selecting from the animal's rich sensory input a small subset of goal-relevant information for further processing, at the expense of other less useful information, which is largely unprocessed and ignored. Knudsen diverges from van Boxtel and colleagues, however, in discussing the endpoint of attentional filtering: in this case, Knudsen agrees with Bundesen and colleagues that attention is fundamentally responsible for furnishing working memory with its specific content (Knudsen, 2007). Knudsen's framework for attention involves four component processes: working memory, competitive selection, based on biased competition (Desimone and Duncan, 1995), top-down sensitivity control, and automatic bottom-up saliency filtering (Knudsen, 2007). All of these processes interact to make up what is commonly referred to as attention.

Given that working memory is by definition a conscious process, this framework suggests that attention, as a compound set of highly interconnected functions, is very closely aligned with consciousness. One interpretation of this expanded view, following arguments from a range of authors (Baars, 2005; Dehaene and Changeux, 2011) is that attention, whether top-down or bottomup, acts as the selecting mechanism for conscious contents, while the working memory component acts as the specific store of (at least some) conscious detail (see Figure 2). This perspective is in accord with Dehaene and Changeux's (2011) global neuronal workspace theory, which sees attention as "serving as a 'gateway' that regulates which information reaches conscious processing" and that "withdrawal of attentional selection is used to modulate conscious access." In addition, the concept of a "global workspace" of conscious access bears many similarities to working memory (Baars, 2005).

\section{EVIDENCE FOR LINKS BETWEEN ATTENTION AND CONSCIOUSNESS}

Considerable evidence exists from multiple domains supporting a tight link between attention and consciousness. For instance, a range of behavioral paradigms demonstrate that a reduction in attentional focus towards a given stimulus also leads to a reduced likelihood in consciously detecting the stimulus. Inattentional blindness, coined by Mack and Rock (1998), refers to the failure to consciously detect an unexpected, but otherwise perfectly visible stimulus, when attention is engaged elsewhere. In the classic paradigm, observers have to decide which of the vertical or horizontal line of a briefly presented central cross is the longest. On crucial trials, an additional shape appears near the cross, and after the trial participants are asked whether they detected the shape. Most fail to notice it. In a more dramatic version of this experiment, approximately half of all participants fail to spot a person in a gorilla suit who unexpectedly walks across the screen in a video, when attention is devoted to a difficult task of counting basketball passes by players wearing white shirts while ignoring passes by players wearing black shirts (Simons and Chabris, 1999). The study by Cohen et al. (2011), described earlier, is another example of such a paradigm.

A related paradigm, change blindness, involves an inability to be conscious of a change in a visual scene, as long as attention is not directed to the critical changing detail (Rensink et al., 1997). For instance, if two otherwise identical images of an outdoor café scene between two people are alternated every $640 \mathrm{~ms}$, separated by a blank screen, participants will fail to be conscious of a radical shift in the position of a prominent background railing for on average 


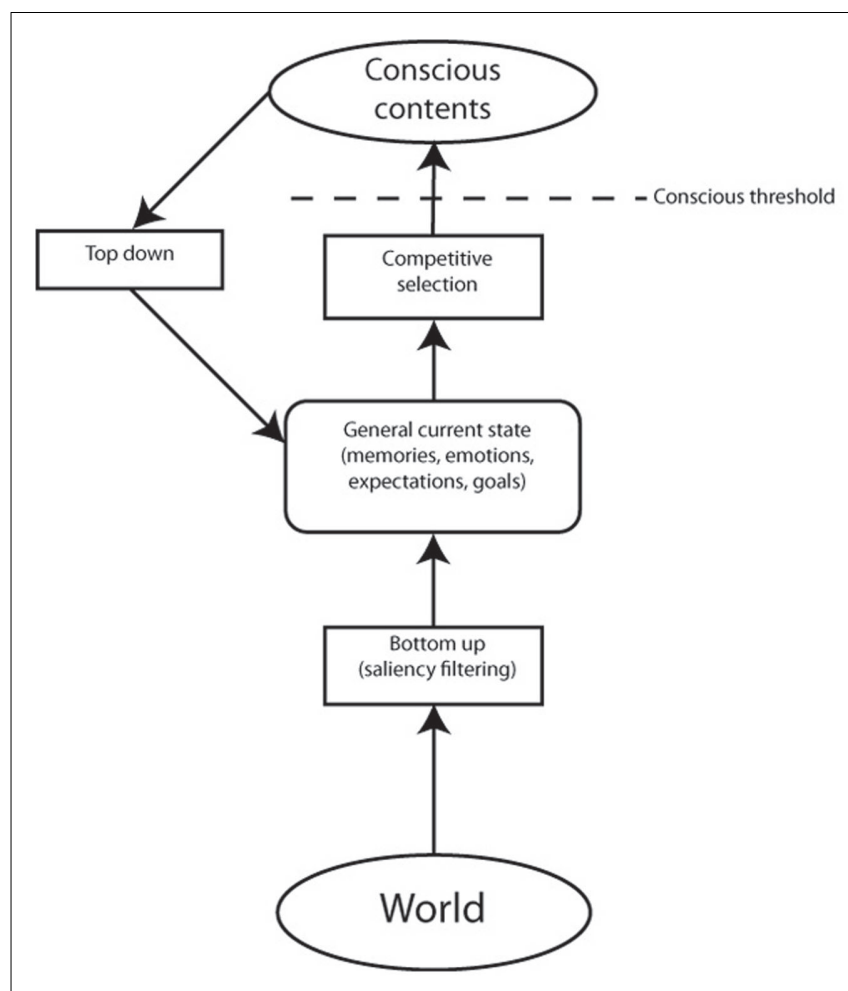

FIGURE 2 |Adaptation of model of attention as formulated by Knudsen (2007) to incorporate consciousness. Bottom-up attention initially processes sensory input according to low level and biologically salient filters. This data is then compared with long-term memory representations, current goals, and other internal states, in order to assign weightings for an attentional competitive selection process. The result of this process leads to the strongest signals being favorably activated, with others inhibited. Those items gaining sufficient excitation will enter consciousness. Conscious contents, which are maintained in working memory in the model, can also be influenced by a recurrent loop with top-down attention biasing weightings for competitive selection. According to this model, it is possible for both top-down and bottom-up attention to occur for unconscious items (for instance by modulating expectations without the object of those expectations having sufficient strength in the subsequent competitive selection process to reach consciousness). However, conscious access for a given object is only possible following an attentional competitive selection process, which can be modulated by top-down and/or bottom-up weightings.

10 s. Interestingly, participants more readily notice a change under these conditions if it alters the functional relationship between semantically central items in the visual scene, as compared to a visually equivalent irrelevant change (Sampanes et al., 2008). Presumably, this form of change is more likely to attract attention, which then ameliorates the change blindness effect.

Particularly relevant to exploring links between attention and consciousness are neurological cases of hemispatial neglect, which are most often considered as reflecting attentional deficits (Mesulam, 1981, 1999; Husain et al., 1997). Historically, neglect has been most closely linked with damage to right posterior parietal cortex, however more recent evidence suggests a substantial proportion of neglect patients instead have right lateral prefrontal damage (Husain and Kennard, 1996; Husain and Rorden, 2003). Neglect is a condition signified by a marked reduction in awareness of one side of space, usually on the left (although the syndrome is equally commonly described as an impairment in being able to attend to one side of space). Unlike blindsight, which is limited to vision, neglect symptoms appear to impact all senses, including touch (Sarri et al., 2006), although perceptual tests confirm that the condition is not one of sensory impairment per se. Functional imaging studies in neglect patients have further demonstrated the link between consciousness and the PPN. For example, in one study, a neglect patient failed to notice a left finger touch on half the trials because of concurrent visual stimulation on the right. Activation was observed in the intact portions of the PPN only when the patient was aware of the touch (Sarri et al., 2006). Complementing the patient data, one intriguing study in normal participants simulated the symptoms of neglect: conscious detection rates for left sided targets were significantly reduced following TMS applied to right posterior parietal cortex (Muggleton et al., 2006).

These observations indicate that neglect as a syndrome is associated with the lateral prefrontal and posterior parietal regions, and by exhibiting symptoms which could easily be formulated either as attentional or as consciousness deficits, neglect reinforces the view that these two processes are closely connected.

\section{WHAT IS THE FUNCTIONAL ROLE OF THE PREFRONTAL PARIETAL NETWORK?}

Prefrontal parietal network activity is consistently reported in both attention and consciousness studies (Rees, 2007). However, as mentioned above, current views of attention describe it as a multifaceted process, closely connected to working memory, as well as executive control, which are all collectively deployed in order to carry out potentially complex goals.

Investigation into the functional role of the PPN has a long history, and has centered on working memory and executive processes (Wager and Smith, 2003). PFC lesions in particular have for many decades been associated with working memory and organizational deficits. For instance, Bor et al. (2006) used the spatial span task to demonstrate that the extent of frontal lobe damage was related to the size of working memory deficit, with those patients who had damage to the right DLPFC particularly impaired.

Prefrontal parietal network activity, however, can be found for almost any demanding or novel task (Miller and Cohen, 2001; Duncan, 2006) raising the possibility that high-level cognitive functions and consciousness may be intrinsically associated. In accord with this suggestion, studies have shown that conscious engagement is required for the use of most logical operations, assimilating information based on cause and effect, the encoding of almost all forms of stimulus sequences, counting and other non-trivial mathematic tasks, as well as for the acquisition and deployment of most social and cultural forms of knowledge (Baumeister and Masicampo, 2010). An intriguing corollary of the link between consciousness and novel or complex task processing is the robust finding that practice and automaticity reduces cognitive demand, prefrontal parietal activity, and awareness of details of a task (Jenkins et al., 1994; Landmann et al., 2007).

It should be noted, however, that rare instances of relatively sophisticated unconscious processing have also been reported, 
for instance involving a speeded conscious recognition of a semantically incongruent scene (Mudrik et al., 2011) or a modulation of response to unconscious no-go signals in a go no-go paradigm (van Gaal et al., 2008). Neither of these examples, however, involve the processing of a novel series of data, which even in simple forms appears to require consciousness. Indeed, when target letters or monotonic sounds are rapidly presented in sequence, conscious detection, and effective performance of any targets aside from the initial one may be delayed or even abolished. Intriguingly, late prefrontal MEG signatures corresponding to target detection are also delayed or abolished in line with performance impairment (Marti et al., 2012).

Dijksterhuis et al. (2006) have reported an additional unconscious advantage effect, where the integration of a large set of data is performed better when unconsciously processed, for instance in a ranking task between four imaginary cars, each having 12 facts revealed about them. However, more recent attempts to replicate this finding have found either no effect, or a clear conscious advantage effect instead, raising doubt on the consistency of this finding (Newell et al., 2009; Aczel et al., 2011).

Examining how consciousness relates to other high-level cognitive processes may provide an important avenue for exploring the mechanism and details of conscious function. One approach in this context is to ask which processes most robustly activate the PPN. One recent fMRI study has suggested that PPN activity does not simply reflect task difficulty (Bor and Owen, 2007). Bor and Owen (2007) compared PPN activity for a set of highlevel tasks: a standard random digit span working memory task, two structured digit span tasks where participants could improve performance using memory-based or novel mathematical chunking techniques, a long-term memory retrieval task and a mental arithmetic task. Despite the fact that the structured versions of the digit span tasks reduced task demands due to the strategic chunking of the stimuli, these conditions activated the PPN significantly more than all the other conditions, with the mathematical chunking condition activating PPN most robustly of all. In other words, detecting and utilizing regular patterns activates the PPN more robustly than working memory, long-term memory, or mental arithmetic alone - even though these tasks were equally or more difficult, according to accuracy scores. Other studies have also shown that the PPN is closely associated with similar recoding processes, including when compared with more demanding versions of the same task (Prabhakaran et al., 2000; Bor et al., 2001, 2003, 2004; Moore et al., 2006; Bor and Owen, 2007; Murray and Ranganath, 2007).

Therefore, within the general context of using consciousness to carry out complex or novel goals, there may be a special role for chunking processes, which search for, discover and exploit patterns in order to optimize task performance. The special role that chunking may play in consciousness links in closely with the critical need for conscious involvement in serial forms of data and aligns with the serial nature of phenomenal experience (Seth et al., 2005).

This perspective suggests a possible functional role for consciousness: when a given automatic, unconscious routine is either not optimal, or even detrimental (in the sense that it increases the likelihood of the animal not meeting its biological needs, or of putting it in danger), usually due to novel or complex challenges, the relevant signals would be integrated into ongoing conscious contents in order to facilitate discovery of an innovative solution out of this impasse. Solutions, which may require sophisticated forms of learning and flexible behavior, are more likely within consciousness because only here can disparate forms of data be integrated in working memory, after attention has prioritized the processing of this data. The detection and use of "chunked" data patterns within a stimulus stream, or relating to motor output sequences, would be an especially powerful means of discovering such innovative solutions, since chunking can lower memory demands by data compression, and via regularity detection can uncover profound, mechanistic features of the environment. Chunking greatly facilitates automatization, which in turn reduces the burden on conscious contents so that other complex or novel tasks could gain conscious access and benefit from further optimization.

Although this process is likely to be metabolically expensive, requiring activity within a large cortical network, this view of consciousness is nevertheless evolutionarily valuable because metabolic costs can in other ways be reduced, by more efficiently streamlining many tasks, and by discovering novel techniques to intelligently avoid complex threats or obtain challenging rewards.

\section{CONCLUSION}

There is compelling evidence that at least some of the "core" correlates of consciousness reside in the PPN. Many current neurobiological theories of consciousness reflect this view, either explicitly or implicitly, in their models. Despite this, such theories tend not to dwell on the psychological and cognitive processes that the PPN may support; these include, prominently, attention, working memory, and chunking. Indeed, the attentional functions supported by the PPN have been argued to be dissociable from the core processes underlying consciousness.

Here, we have argued that these dissociations have been overstated. The relevant empirical evidence is open to alternative explanations, and two critical issues complicate any interpretation in favor of a double dissociation: (i) the way that attention is defined theoretically (e.g., as bottom-up, top-down, or both) and (ii) how attentional deployment can be adequately controlled for empirically. An alternative view, following (Rees and Lavie, 2001; Baars, 2005), among others, is that attention is intimately linked with consciousness, and may be best understood as involved in the selection of specific conscious contents. On this view, some form of attention is necessary for consciousness, although attention (without working memory) is not sufficient for consciousness.

Attention is best viewed as part of an integrated set of processes, including working memory, whose purpose is to achieve complex or novel goals. Activity within the PPN is associated both with consciousness and with this flexible set of processes, and is especially prominent if the task involves the chunking or recoding of information into regular patterns in order to optimize performance and reduce demands. Chunking is one of the most profound learning mechanisms available for human cognition (Ericcson et al., 1980; Gobet et al., 2001), and thus a powerful means by which 
integrated information is "reshaped" in the brain. Therefore, further investigations about the role that chunking, as well as attention and working memory, plays in consciousness might prove highly useful in understanding the psychological features of consciousness and their relation to underlying neural mechanisms. More generally, consciousness science is likely to benefit from a broader consideration of the functional role of the PPN including, and

\section{REFERENCES}

Abe, M., Hanakawa, T., Takayama, Y., Kuroki, C., Ogawa, S., and Fukuyama, H. (2007). Functional coupling of human prefrontal and premotor areas during cognitive manipulation. J. Neurosci. 27, 3429-3438.

Aczel, B., Lukacs, B., Komlos, J., and Aitken, M. R. F. (2011). Unconscious intuition or conscious analysis? Critical questions for the Deliberation-Without-Attention paradigm. Judgm. Decis. Mak. 6, 351-358.

Alais, D., van Boxtel, J. J., Parker, A., and van Ee, R. (2010). Attending to auditory signals slows visual alternations in binocular rivalry. Vision Res. 50, 929-935.

Baars, B. J. (1988). A cognitive theory of consciousness. New York, NY: Cambridge University Press.

Baars, B. J. (2005). Global workspace theory of consciousness: toward a cognitive neuroscience of human experience. Prog. Brain Res. 150, 45-53.

Baars, B. J., Ramsoy, T. Z., and Laureys, S. (2003). Brain, conscious experience and the observing self. Trends Neurosci. 26, 671-675.

Baddeley, A. D., and Hitch, G. (1974). "Working memory," in The Psychology of Learning and Motivation, ed. G. A. Bower (New York, NY: Academic Press), 47-89.

Balduzzi, D., and Tononi, G. (2008). Integrated information in discrete dynamical systems: motivation and theoretical framework. PLoS Comput. Biol. 4, e1000091. doi:10.1371/journal.pcbi.1000091

Barrett, A. B., and Seth, A. K. (2011). Practical measures of integrated information for time-series data. PLoS Comput. Biol. 7, e1001052. doi:10.1371/journal.pcbi.1001052

Baumeister, R. F., and Masicampo, E. J. (2010). Conscious thought is for facilitating social and cultural interactions: how mental simulations serve the animal-culture interface. Psychol. Rev. 117, 945-971.

Beck, D. M., Muggleton, N., Walsh, V., and Lavie, N. (2006). Right parietal cortex plays a critical role in change blindness. Cereb. Cortex 16, 712-717.
Block, N. (2007). Consciousness, accessibility, and the mesh between psychology and neuroscience. Behav. Brain Sci. 30, 481-499.

Bor, D., Cumming, C. N, Scott, E., and Owen, A. M. (2004). Prefrontal cortical involvement in verbal encoding strategies. Eur. J. Neurosci. 19, 3365-3370.

Bor, D., Duncan, J., Lee, A. C., Parr, A., and Owen, A. M. (2006). Frontal lobe involvement in spatial span: Converging studies of normal and impaired function. Neuropsychologia 44, 229-237.

Bor, D., Duncan, J., and Owen, A. M. (2001). The role of spatial configuration in tests of working memory explored with functional neuroimaging. Scand. J. Psychol. 42, 217-224.

Bor, D., Duncan, J., Wiseman, R. J., and Owen, A. M. (2003). Encoding strategies dissociate prefrontal activity from working memory demand. Neuron 37, 361-367.

Bor, D., and Owen, A. M. (2007). A common prefrontal-parietal network for mnemonic and mathematical recoding strategies within working memory. Cereb. Cortex 17, 778-786.

Boyer, J., and Ro, T. (2007). Attention attenuates metacontrast masking. Cognition 104, 135-149.

Bressan, P., and Pizzighello, S. (2008). The attentional cost of inattentional blindness. Cognition 106, 370-383. Communication. London: Pergamon Press.

Bundesen, C. (1990). A theory of visual attention. Psychol. Rev. 97, 523-547.

Bundesen, C., Habekost, T., and Kyllingsbaek, S. (2005). A neural theory of visual attention: bridging cognition and neurophysiology. Psychol. Rev. 112, 291-328.

Bussche, E. V., Hughes, G., Humbeeck, N. V., and Reynvoet, B. (2010). The relation between consciousness and attention: an empirical study using the priming paradigm. Conscious. Cogn. 19, 86-97.

Cabeza, R., and Nyberg, L. (2000). Imaging cognition II: An empirical review of 275 PET and fMRI studies. J. Cogn. Neurosci. 12, 1-47.
Broadbent, D. E. (1958). Perception and

indeed exploiting, its intimate links to attention, working memory, and chunking.

\section{ACKNOWLEDGMENTS}

Daniel Bor and Anil K. Seth gratefully acknowledge support from the Dr. Mortimer and Theresa Sackler Foundation. Anil K. Seth is also supported by EPRSC Leadership Fellowship EP/G007543/1.

Carmel, D., Lavie, N., and Rees, G. (2006). Conscious awareness of flicker in humans involves frontal and parietal cortex. Curr. Biol. 16, 907-911.

Carrasco, M., Ling, S., and Read, S. (2004). Attention alters appearance. Nat. Neurosci. 7, 308-313.

Carrasco, M., Penpeci-Talgar, C., and Eckstein, M. (2000). Spatial covert attention increases contrast sensitivity across the CSF: support for signal enhancement. Vision Res. 40, 1203-1215.

Chelazzi, L., Duncan, J., Miller, E. K., and Desimone, R. (1998). Responses of neurons in inferior temporal cortex during memory-guided visual search. J. Neurophysiol. 80, 2918-2940.

Cohen, M. A., Alvarez, G. A., and Nakayama, K. (2011). Natural-scene perception requires attention. Psychol. Sci. 22, 1165-1172.

Corbetta, M., Shulman, G. L., Miezin, F. M., and Petersen, S. E. (1995). Superior parietal cortex activation during spatial attention shifts and visual feature conjunction. Science 270, 802-805.

Davis, M. H., Coleman, M. R., Absalom, A. R., Rodd, J. M., Johnsrude, I. S., Matta, B. F., Owen, A. M., and Menon, D. K. (2007). Dissociating speech perception and comprehension at reduced levels of awareness. Proc. Natl. Acad. Sci. U.S.A. 104, 16032-16037.

de Gardelle, V., and Kouider, S. (2009). "Cognitive theories of consciousness," in Encyclopedia of Consciousness, ed. W. Banks (Oxford: Academic Press), 135-146.

Dehaene, S., and Changeux, J. P. (2011). Experimental and theoretical approaches to conscious processing. Neuron 70, 200-227.

Dehaene, S., and Naccache, L. (2001). Towards a cognitive neuroscience of consciousness: basic evidence and a workspace framework. Cognition 79, 1-37.

Dehaene, S., Naccache, L., Cohen, L. Bihan, D. L., Mangin, J. F., Poline, J. B., and Riviere, D. (2001). Cerebral mechanisms of word masking and unconscious repetition priming. Nat. Neurosci. 4, 752-758.
Del Cul, A., Dehaene, S., Reyes, P., Bravo, E., and Slachevsky, A (2009). Causal role of prefrontal cortex in the threshold for access to consciousness. Brain 132(Pt 9), 2531-2540.

Desimone, R., and Duncan, J. (1995). Neural mechanisms of selective visual attention. Annu. Rev. Neurosci. 18, 193-222.

Diaz, M. T., and McCarthy, G. (2007). Unconscious word processing engages a distributed network of brain regions. J. Cogn. Neurosci. 19, 1768-1775.

Dijksterhuis, A., Bos, M. W., Nordgren, L. F., and van Baaren, R. B. (2006). On making the right choice: the deliberation-without-attention effect. Science 311, 1005-1007.

Duncan, J. (2006). EPS Mid-Career Award 2004: brain mechanisms of attention. Q. J. Exp. Psychol. (Colchester) 59, 2-27.

Edelman, G. M. (1989). The remembered present. New York, NY: Basic Books.

Edelman, G. M. (2003). Naturalizing consciousness: a theoretical framework. Proc. Natl. Acad. Sci. U.S.A. 100, 5520-5524.

Edelman, G. M., Gally, J. A., and Baars, B. J. (2011). Biology of consciousness. Front. Psychol. 2:4. doi:10.3389/fpsyg.2011.00004

Ericcson, K. A., Chase, W. G., and Falloon, S. (1980). Acquisition of a memory skill. Science 208, 1181-1182.

Erickson, K. I., Colcombe, S. J., Wadhwa, R., Bherer, L., Peterson, M. S. Scalf, P. E., Kim, J. S., Alvarado, M., and Kramer, A. F. (2007) Training-induced functional activation changes in dual-task processing: an FMRI study. Cereb. Cortex 17, 192-204.

Eriksson, J., Larsson, A., Riklund Åhlström, K., and Nyberg, L. (2004). Visual consciousness: dissociating the neural correlates of perceptual transitions from sustained perception with fMRI. Conscious. Cogn. 13, 61-72.

Ffytche, D. H., and Zeki, S. (2011). The primary visual cortex, and feedback to it, are not necessary for conscious vision. Brain 134(Pt 1): 247-257. 
Fisch, L., Privman, E., Ramot, M., Harel, M., Nir, Y., Kipervasser, S., Andelman, F., Neufeld, M. Y., Kramer, U., Fried, I., and Malach, R. (2009). Neural ignition: enhanced activation linked to perceptual awareness in human ventral stream visual cortex. Neuron 64, 562-574.

Fleming, S. M., Weil, R. S., Nagy, Z., Dolan, R. J., and Rees, G. (2010). Relating introspective accuracy to individual differences in brain structure. Science 329, 1541-1543.

Gaillard, R., Dehaene, S., Adam, C., Clemenceau, S., Hasboun, D., Baulac, M., Cohen, L., and Naccache, L. (2009). Converging intracranial markers of conscious access. PLoS Biol. 7, e61. doi:10.1371/journal.pbio. 1000061

Gobet, F., Lane, P. C. R., Croker, S., Cheng, P. C-H., Jones, G., Oliver, L., and Pine, J. M. (2001). Chunking mechanisms in human learning. Trends Cogn. Sci. (Regul. Ed.) 5, 236-243.

Hasson, U., Skipper, J. I., Nusbaum, H. C., and Small, S. L. (2007). Abstract coding of audiovisual speech: beyond sensory representation. Neuron 56, 1116-1126.

Haynes, J. D., Deichmann, R., and Rees, G. (2005). Eye-specific effects of binocular rivalry in the human lateral geniculate nucleus. Nature 438 , 496-499.

Hipp, J. F., Engel, A. K., and Siegel, M. (2011). Oscillatory synchronization in large-scale cortical networks predicts perception. Neuron 69, 387-396.

Huang, Y. Z., Edwards, M. J., Rounis, E., Bhatia, K. P., and Rothwell, J. C. (2005). Theta burst stimulation of the human motor cortex. Neuron 45 , 201-206.

Husain, M., and Kennard, C. (1996). Visual neglect associated with frontal lobe infarction. J. Neurol. 243, 652-657.

Husain, M., and Rorden, C. (2003). Non-spatially lateralized mechanisms in hemispatial neglect. Nat. Rev. Neurosci. 4, 26-36.

Husain, M., Shapiro, K., Martin, J., and Kennard, C. (1997). Abnormal temporal dynamics of visual attention in spatial neglect patients. Nature 385 , 154-156.

Jenkins, I. H., Brooks, D. J., Nixon, P. D., Frackowiak, R. S., and Passingham, R. E. (1994). Motor sequence learning: a study with positron emission tomography. J. Neurosci. 14, 3775-3790.

Joseph, J. S., Chun, M. M., and Nakayama, K. (1997). Attentional requirements in a 'preattentive' feature search task. Nature 387, 805-807.

Kanai, R., Bahrami, B., and Rees, G. (2010). Human Parietal Cortex Structure Predicts Individual Differences in Perceptual Rivalry. Curr. Biol. 20, 1626-1630.

Kentridge, R. W., Nijboer, T. C., and Heywood, C. A. (2008). Attended but unseen: visual attention is not sufficient for visual awareness. Neuropsychologia 46, 864-869.

Kleinschmidt, A., Buchel, C., Zeki, S., and Frackowiak, R. S. (1998). Human brain activity during spontaneously reversing perception of ambiguous figures. Proc. Biol. Sci. 265, 2427-2433.

Knapen, T., Brascamp, J., Pearson, J., van Ee, R., and Blake, R. (2011). The role of frontal and parietal brain areas in bistable perception. J. Neurosci. 31, 10293-10301.

Knight, R. T., and Grabowecky, M. (1995). "Escape from linear time: prefrontal cortex and conscious experience," in The New Cognitive Neurosciences, ed. M. Gazzaniga (Massachusetts: MIT Press), 1357-1371.

Knudsen, E. I. (2007). Fundamental components of attention. Annu. Rev. Neurosci. 30, 57-78.

Koch, C., and Tsuchiya, N. (2007). Attention and consciousness: two distinct brain processes. Trends Cogn. Sci. (Regul. Ed.) 11, 16-22.

Koechlin, E., and Summerfield, C. (2007). An information theoretical approach to prefrontal executive function. Trends Cogn. Sci. (Regul. Ed.) 11, 229-235.

Kouider, S. (2009). "Neurobiological theories of consciousness," in Encyclopedia of Consciousness, ed. W. Banks (Oxford: Academic Press), 87-100.

Kouider, S., de Gardelle, V., Sackur, J., and Dupoux, E. (2010). How rich is consciousness? The partial awareness hypothesis. Trends Cogn. Sci. (Regul. Ed.) 14, 301-307.

Lamme, V. A. (2006). Towards a true neural stance on consciousness. Trends Cogn. Sci. (Regul. Ed.) 10, 494-501.

Lamme, V. A. F. (2010). How neuroscience will change our view on consciousness. Cogn. Neurosci. 1, 204220.

Landmann, C., Dehaene, S., Pappata, S., Jobert, A., Bottlaender, M., Roumenov, D., and Le Bihan, D. (2007). Dynamics of prefrontal and cingulate activity during a reward-based logical deduction task. Cereb. Cortex 17, 749-759.
Lau, H., and Rosenthal, D. (2011). Empirical support for higher-order theories of conscious awareness. Trends Cogn. Sci. (Regul. Ed.) 15, 365-373.

Lau, H. C., and Passingham, R. E. (2006). Relative blindsight in normal observers and the neural correlate of visual consciousness. Proc. Natl. Acad. Sci. U.S.A. 103, 18763-18768.

Laureys, S., Faymonville, M. E., Luxen, A., Lamy, M., Franck, G., and Maquet, P. (2000). Restoration of thalamocortical connectivity after recovery from persistent vegetative state. Lancet 355, 1790-1791.

Lee, S. H., Blake, R., and Heeger, D. J. (2005). Traveling waves of activity in primary visual cortex during binocular rivalry. Nat. Neurosci. 8 , 22-23.

Li, F. F., VanRullen, R., Koch, C., and Perona, P. (2002). Rapid natural scene categorization in the near absence of attention. Proc. Natl. Acad. Sci. U.S.A. 99, 9596-9601.

Lumer, E. D., Friston, K. J., and Rees, G. (1998). Neural correlates of perceptual rivalry in the human brain. Science 280, 1930-1934.

Lumer, E. D., and Rees, G. (1999). Covariation of activity in visual and prefrontal cortex associated with subjective visual perception. Proc. Natl. Acad. Sci. U.S.A. 96 1669-1673.

Luo, Q., Mitchell, D., Cheng, X., Mondillo, K., McCaffrey, D., Holroyd, T., Carver, F., Coppola, R., and Blair, J. (2009). Visual awareness, emotion, and gamma band synchronization. Cereb. Cortex 19, 1896-1904.

Lycan, W. G. (2004). "The superiority of HOP over HOT," in Higher-Order Theories of Consciousness: An Anthol ogy, ed. R. J. Gennaro (Amsterdam: John-Benjamins), 93-113.

Mack, A., and Rock, I. (1998). Inattentional Blindness. Cambridge, MA: MIT Press.

Marti, S., Sigman, M., and Dehaene, S. (2012). A shared cortical bottleneck underlying Attentional Blink and Psychological Refractory Period. Neuroimage 59, 2883-2898.

Melloni, L., Molina, C., Pena, M., Torres, D., Singer, W., and Rodriguez, E. (2007). Synchronization of neural activity across cortical areas correlates with conscious perception. $J$. Neurosci. 27, 2858-2865.

Mesulam, M. M. (1981). A cortical network for directed attention and unilateral neglect. Ann. Neurol. 10, 309-325.

Mesulam, M.-M. (1999). Spatial attention and neglect: parietal, frontal and cingulate contributions to the mental representation and attentional targeting of salient extrapersonal events. Philos. Trans. R. Soc. Lond. B Biol. Sci. 354, 1325-1346.

Miller, E. K., and Cohen, J. D. (2001). An integrative theory of prefrontal cortex function. Annu. Rev. Neurosci. 24, 167-202.

Modha, D. S., and Singh, R. (2010) Network architecture of the longdistance pathways in the macaque brain. Proc. Natl. Acad. Sci. U.S.A. 107, 13485-13490.

Moore, C. D., Cohen, M. X., and Ranganath, C. (2006). Neural mechanisms of expert skills in visual working memory. J. Neurosci. 26, 11187-11196.

Mudrik, L., Breska, A., Lamy, D., and Deouell, L. Y. (2011). Integration without awareness: expanding the limits of unconscious processing. Psychol. Sci. 22, 764-770.

Muggleton, N. G., Postma, P., Moutsopoulou, K., Nimmo-Smith, I., Marcel, A., and Walsh, V. (2006). TMS over right posterior parietal cortex induces neglect in a scenebased frame of reference. Neuropsychologia 44, 1222-1229.

Murray, L. J., and Ranganath, C. (2007) The dorsolateral prefrontal cortex contributes to successful relational memory encoding. J. Neurosci. 27 , 5515-5522.

Newell, B. R., Wong, K. Y., and Cheung, J. C. H. (2009). Think, blink or sleep on it? The impact of modes of thought on complex decision making. Q. J. Exp. Psychol. 62, 707-732.

Norman, D. A., and Shallice, T. (1986).

"Willed and automatic control of behavior," in Consciousness and SelfRegulation. Advances in Research and Theory, eds R. J. Davidson, G. E. Schwarts, and D. Shapiro (New York: Plenum Press), 1-18.

Paffen, C. L., Alais, D., and Verstraten, F. A. (2006). Attention speeds binocular rivalry. Psychol. Sci. 17, 752-756.

Posner, M. I. (1994). Attention: the mechanisms of consciousness. Proc. Natl. Acad. Sci. U.S.A. 91, 7398-7403.

Prabhakaran, V., Narayanan, K., Zhao, Z., and Gabrieli, J. D. (2000). Integration of diverse information in working memory within the frontal lobe. Nat. Neurosci. 3, 85-90.

Ramachandran, V. S., and Cobb, S. (1995). Visual attention modulates metacontrast masking. Nature 373 66-68.

Rees, G. (2007). Neural correlates of the contents of visual awareness in humans. Philos. Trans. 
R. Soc. Lond. B Biol. Sci. 362, $877-886$.

Rees, G., and Lavie, N. (2001). What can functional imaging reveal about the role of attention in visual awareness? Neuropsychologia 39, 1343-1353.

Rensink, R. A., O’Regan, J. K., and Clark, J. J. (1997). To see or not to see: The need for attention to perceive changes in scenes. Psychol. Sci. 8, 368-373.

Rosenthal, D. M. (2005). Consciousness and Mind. Oxford: Clarendon Press.

Rounis, E., Maniscalco, B., Rothwell, J. C., Passingham, R. E., and Lau, H. (2010). Theta-burst transcranial magnetic stimulation to the prefrontal cortex impairs metacognitive visual awareness. Cogn. Neurosci. 1, $165-175$.

Ruff, C. C., Kristjansson, A., and Driver, J. (2007). Readout from iconic memory and selective spatial attention involve similar neural processes. Psychol. Sci. 18, 901-909.

Sadaghiani, S., Hesselmann, G., and Kleinschmidt, A. (2009). Distributed and antagonistic contributions of ongoing activity fluctuations to auditory stimulus detection. $\mathrm{J}$. Neurosci. 29, 13410-13417.

Sampanes, A. C., Tseng, P., and Bridgeman, B. (2008). The role of gist in scene recognition. Vision Res. 48, 2275-2283.

Sarri, M., Blankenburg, F., and Driver, J. (2006). Neural correlates of crossmodal visual-tactile extinction and of tactile awareness revealed by fMRI in a right-hemisphere stroke patient. Neuropsychologia 44, 2398-2410.

Seth, A. (2007). Models of Consciousness. Scholarpedia 2007. Available at: http://www.scholarpedia.org/article/ Models_of_consciousness

Seth, A. (2009). Explanatory correlates of consciousness: Theoretical and computational challenges. Cogn. Comput. 1, 50-63.

Seth, A. K. (2005). Causal connectivity of evolved neural networks during behavior. Network 16, 35-54.

Seth, A. K. (2010). The grand challenge of consciousness. Front. Psychol. 1:5. doi:10.3389/fpsyg.2010.00005

Seth, A. K., Baars, B. J., and Edelman, D. B. (2005). Criteria for consciousness in humans and other mammals. Conscious. Cogn. 14, 119-139.

Seth, A. K., Barrett, A. B., and Barnett, L. (2011). Causal density and integrated information as measures of conscious level. Philos. Transact. R. Soc. A Math. Phys. Eng. Sci. 369, 3748-3767.

Seth, A. K., Dienes, Z., Cleeremans, A., Overgaard, M., and Pessoa, L. (2008). Measuring consciousness: relating behavioural and neurophysiological approaches. Trends Cogn. Sci. (Regul. Ed.) 12, 314-321.

Shelley-Tremblay, J., and Mack, A. (1999). Metacontrast masking and attention. Psychol. Sci. 10, 508-515.

Sillito, A. M., Cudeiro, J., and Jones, H. E. (2006). Always returning: feedback and sensory processing in visual cortex and thalamus. Trends Neurosci. 29, 307-316.

Simons, D. J., and Chabris, C. F. (1999). Gorillas in our midst: sustained inattentional blindness for dynamic events. Perception 28, 1059-1074.

Simons, J. S., Peers, P. V., Mazuz, Y. S., Berryhill, M. E., and Olson, I. R. (2010). Dissociation between memory accuracy and memory confidence following bilateral parietal lesions. Cereb. Cortex 20, 479-485.

Sterzer, P., Russ, M. O., Preibisch, C., and Kleinschmidt, A. (2002). Neural correlates of spontaneous direction reversals in ambiguous apparent visual motion. Neuroimage 15, 908-916.

Tapia, E., Breitmeyer, B. G., and Shooner, C. R. (2010). Role of taskdirected attention in nonconscious and conscious response priming by form and color. J. Exp. Psychol. Hum. Percept. Perform. 36, 74-87.

Tata, M. S., and Giaschi, D. E. (2004). Warning: attending to a mask may be hazardous to your perception. Psychon. Bull. Rev. 11, 262-268.

Theeuwes, J., Kramer, A. F., and Atchley, P. (1999). Attentional effects on preattentive vision: spatial precues affect the detection of simple features. J. Exp. Psychol. Hum. Percept. Perform. 25, 341-347.

Thorpe, S., Fize, D., and Marlot, C. (1996). Speed of processing in the human visual system. Nature 381, 520-522.

Tononi, G. (2008). Consciousness as integrated information: a provisional manifesto. Biol. Bull. 215, 216-242.

Tononi, G., and Edelman, G. M. (1998). Consciousness and complexity. Science 282, 1846-1851.

Tononi, G., and Koch, C. (2008). The neural correlates of consciousness: an update. Ann. N. Y. Acad. Sci. 1124, 239-261.

Tshibanda, L., Vanhaudenhuyse, A., Galanaud, D., Boly, M., Laureys, S. and Puybasset, L. (2009). Magnetic resonance spectroscopy and diffusion tensor imaging in coma survivors: promises and pitfalls. Prog. Brain Res. 177, 215-229.

Tsubomi, H., Ikeda, T., Hanakawa, T., Hirose, N., Fukuyama, H., and Osaka, N. (2011). Dissociable neural activations of conscious visibility and attention. J. Cogn. Neurosci. $1-11$.

Turatto, M., Sandrini, M., and Miniussi, C. (2004). The role of the right dorsolateral prefrontal cortex in visual change awareness. Neuroreport 15, 2549-2552.

van Boxtel, J. J. A., Tsuchiya, N., and Koch, C. (2010a). Opposing effects of attention and consciousness on afterimages. Proc. Natl. Acad. Sci. U.S.A. 107, 8883-8888.

van Boxtel, J. J. A., Tsuchiya, N. and Koch, C. (2010b). Consciousness and attention: On sufficiency and necessity. Front. Psychol. 2:12. doi:10.3389/fpsyg.2010.00217

van Gaal, S., Ridderinkhof, K. R. Fahrenfort, J. J., Scholte, H. S., and Lamme, V. A. (2008). Frontal cortex mediates unconsciously triggered inhibitory control. J. Neurosci. 28, 8053-8062.

Voytek, B., Davis, M., Yago, E., Barcelo, F., Vogel, E. K., and Knight, R. T. (2010). Dynamic neuroplasticity after human prefrontal cortex damage. Neuron 68, 401-408.

Wager, T. D., and Smith, E. E. (2003). Neuroimaging studies of working memory: a meta-analysis. Cogn Affect. Behav. Neurosci. 3, 255-274.

Walker, S., Stafford, P., and Davis, G. (2008). Ultra-rapid categoriza- tion requires visual attention: Scenes with multiple foreground objects. $J$. Vis. 8, 21.1-21.12.

Wyart, V., and Tallon-Baudry, C. (2008). Neural dissociation between visual awareness and spatial attention. $J$. Neurosci. 28, 2667-2679.

Yantis, S., Schwarzbach, J., Serences, J. T., Carlson, R. L., Steinmetz, M. A., Pekar, J. J., and Courtney, S. M. (2002). Transient neural activity in human parietal cortex during spatial attention shifts. Nat. Neurosci. 5, 995-1002.

Zaretskaya, N., Thielscher, A., Logothetis, N. K., and Bartels, A. (2010). Disrupting parietal function prolongs dominance durations in binocular rivalry. Curr. Biol. 20, 2106-2111.

Zeki, S. (2007). A theory of microconsciousness. The Blackwell Companion to Consciousness (Oxford: Blackwell), 580-588.

Zeman, A. (2005). What in the world is consciousness? Prog. Brain Res. 150, $1-10$.

Zhang, P., Jamison, K., Engel, S., He, B., and He, S. (2011). Binocular rivalry requires visual attention. Neuron 71 , 362-369.

Conflict of Interest Statement: The authors declare that the research was conducted in the absence of any commercial or financial relationships that could be construed as a potential conflict of interest.

Received: 17 November 2011; accepted: 18 February 2012; published online: 12 March 2012.

Citation: Bor D and Seth AK (2012) Consciousness and the prefrontal parietal network: insights from attention, working memory, and chunking. Front. Psychology 3:63. doi: 10.3389/fpsyg.2012.00063 This article was submitted to Frontiers in Consciousness Research, a specialty of Frontiers in Psychology.

Copyright (c) 2012 Bor and Seth. This is an open-access article distributed under the terms of the Creative Commons Attribution Non Commercial License, which permits non-commercial use, distribution, and reproduction in other forums, provided the original authors and source are credited. 